\title{
Los desafíos de la coordinación intersectorial: Un análisis de la implementación del programa de Apoyo al Recién Nacido
}

\author{
David Opazo \\ CENABAST, Chile
}

\begin{abstract}
Resumen
Esta investigación analiza la estructura del Programa de Apoyo al Recién Nacido, parte de las prestaciones del Sistema Integral a la Infancia de Chile Crece Contigo, desde una perspectiva intersectorial. Se examina la implementación y la coordinación de Programas intersectoriales en los que participan distintos sectores, tanto gubernamentales como privados. Se concluye que los principales fallos están asociados a una mejora de la gestión del programa y no a cambios en su estructura organizacional. Se recomienda fortalecer los talleres prenatales, contar con personal a nivel de la Red Hospitalaria directamente asociado al Programa e implementar un sistema de registro y gestión que permita superar las brechas de información y fortalecer la totalidad del Programa.
\end{abstract}

Palabras clave: Coordinación intersectorial, implementación de políticas públicas, Desarrollo Infantil Temprano.

\section{The Intersectoral Coordination Challenges: Analysis of the Support Newborn Program Implementation}

\begin{abstract}
This research analyzes the structure, from an intersectoral perspective, of the Programa de Apoyo al Recién Nacido (PARN), part of the benefits of the Integrated System for Children named Chile Crece Contigo. It examines the intersectoral programs implementation and coordination in which various sectors, both governmental and private are involved. The research supports the conclusion that major failures are related to an improvement in PARN management and not to changes in its organizational structure. It is recommended to strengthen the educational module by reinforcing prenatal workshops, having a staff at the hospital network level directly associated with the Program and implementing a registration and management system to overcome information gaps and strengthen the entire Program.
\end{abstract}

Keywords: Intersectoral coordination, public policies implementation, Early Childhood Development.

\section{Los desafíos de la coordinación intersectorial}

Las desigualdades en los primeros años de vida constituyen un aspecto esencial de la superación de la pobreza, en el entendido de que el nacimiento y la infancia forman parte de un período fundamental para el desarrollo y la salud de las personas a lo largo de todo el ciclo de vida.

La multidimensionalidad de las causas que influyen sobre la vulnerabilidad social y la pobreza, im-

\begin{tabular}{l} 
*Dirección de correspondencia [Correspondence \\
address]: \\
David Opazo, CENABAST (Chile) \\
E-mail: dopazo@cenabast.cl \\
\hline
\end{tabular}

pulsan a que las políticas públicas diseñadas para abordar estos fenómenos se aborden desde una perspectiva intersectorial, ya que requieren de relaciones entre distintos sectores para la solución de problemas, suponiendo un un proceso que integra lo político y lo técnico, lo participativo y lo concertado, lo vertical y lo horizontal, con alcances macro, meso y micro.

En Chile, las políticas públicas orientadas a la primera infancia brindan prestaciones de carácter universal, teniendo como horizonte eliminar las desigualdades que se generan en el origen social al momento de nacer. La institucionalización del Programa Chile Crece Contigo (CHCC) como una política pública estable, comprehensiva, intersectorial y de múltiples componentes, considera un conjunto de prestaciones interrelacionadas que se ejecutan a través del Programa Educativo Masivo, el Progra- 
ma de Apoyo al Desarrollo Biopsicosocial, el Programa de Apoyo al Recién Nacido/a y las Prestaciones diferenciales de acuerdo a las características particulares de los niños y niñas. El Programa de Protección Integral a la Infancia de CHCC, tiene un sello multisectorial y orientado a resultados, posee además un diseño de subsistemas para la atención a la primera infancia, brindando cobertura desde la gestación hasta los 4 años de edad, a través del Programa de Apoyo al Desarrollo Biopsicosocial (PADBS) y el Programa de Apoyo al Recién Nacido (PARN).

La implementación de políticas públicas es "en sí misma fuente de problemas complejos que no pueden solucionarse 'ajustándose' mejor a lo planificado o exigiendo a los diseñadores que revisen la tarea" (Cortázar Velarde, 2006); más aún, cuando estas políticas públicas son implementadas intersectorialmente, implican necesariamente interacción entre distintos actores o sectores, con valores, visiones e ideas de la realidad distintos.

De esta forma, el objetivo de la investigación es describir el modelo de gestión, la estructura, los procesos de implementación y la coordinación del Programa de Apoyo al Recién Nacido (PARN), desde una perspectiva intersectorial, buscando identificar y analizar los actuales fallos o cuellos de botella del Programa.

\section{Programas sociales intersectoriales: Los desafíos de la implementación}

Los programas de protección social latinoamericanos han puesto el problema de la intersectorialidad en el primer plano. En la medida en que la pobreza es un problema multidimensional, este debe ser intervenido desde diversos sectores del aparato público. La intersectorialidad es fundamentalmente un proceso político que implica la integración, la inclusividad y la mancomunidad. La cooperación intersectorial es posible mediante la consciencia de que existe un problema social, transversal, que no puede ser resuelto de forma exclusiva por un sector. El fundamento técnico de la intersectoralidad se basa en dos premisas: que la integración entre sectores posibilita la búsqueda de soluciones integrales y que las diferencias entre los distintos sectores pueden resultar productivas para resolver las problemáticas sociales, generando las mejores soluciones (Cunill-Grau, 2014).

Desde el punto de vista conceptual, la coordinación refiere a "la necesidad de asegurar que las distintas organizaciones, públicas y privadas, responsables de la formación de las políticas públicas, trabajen juntas para no producir redundancia ni bre- chas en la entrega de los servicios" (Peters, 1998). Esta necesidad resulta de "la diferenciación de actores por su inserción en esquemas de división del trabajo, ya sea al interior de organizaciones, entre conjuntos organizaciones o entre actores diversos en el plano social" (Echebarría, 2008). Comprende reglas, diseños y procedimientos de tareas dirigidos a lograr la complementariedad y/o la agregabilidad intencionadas de contribuciones y comportamientos de participantes en arreglos sociales multiactorales" (Martínez, 2010). Se pueden distinguir cuatro tipos de sinergias: las cognitivas, las de procesos y actividades, las de recursos, las de autoridad y la cultural o ideológica (Licha y Molina, 2006).

En las políticas públicas intersectoriales, la implementación es una puerta de acceso al aumento de oportunidades en agregación de valor de la política. Esto implica, necesariamente, una interacción estratégica entre distintos actores o sectores, con valores, visiones e ideas de la realidad distintos. El grado de cooperación al que las partes involucradas arriben mediante los continuos procesos de negociación intersectorial, determinarán la capacidad de la política pública para generar valor público (Cortázar Velarde, 2006).

La coordinación puede ser en sentido vertical, ascendente y descendente de la jerarquía; $u$, horizontal, la que propicia dentro del mismo nivel jerárquico la coordinación entre las diversas unidades. De esta forma, las organizaciones tienden a apelar a cuatro mecanismos de coordinación vertical: a través de las referencias jerárquicas, a través de las reglas y planes, a través de una sumatoria de puestos o niveles jerárquicos, y por último, sumando sistemas verticales de información. Para la coordinación horizontal tiende a darse a través del papeleo, el contacto directo, roles vinculantes, equipos de trabajo o la instalación de comités permanentes (Mintzberg, 1988).

\section{El modelo organizacional de Mintz- berg}

El modelo de Mintzberg (1988) es particularmente útil para el estudio de procesos de integración multisectorial. Este visualiza las organizaciones compuestas por seis partes fundamentales: el núcleo operacional, el ápice estratégico, la línea media, la tecno estructura, el staff de soporte y la cultura. Se suman los agentes externos (servicios tercerizados) y el sistema tecnológico y de información, éste último transversal a todas las etapas y actores del proceso de implementación.

Estos componentes mantienen su cohesión mediante mecanismos de coordinación. Mintzberg de- 
fine seis: adaptación mutua, supervisión directa, normalización de procesos, de resultados, de habilidades y normas, y comunicación (Mintzberg, 1988). En la mayoría de las organizaciones, se hacen presentes los distintos mecanismos de coordinación, dando preferencia a uno de ellos. La organización es inducida a diseñarse como una configuración específica: empresarial, maquinal, profesional, diversificada, innovadora, misionera o política.

De esta forma, cualquier organización puede concebirse como el conjunto de todas las formas en que se divide el trabajo en distintas tareas, consiguiendo luego su coordinación (Mintzberg et al., 1997). Este enfoque se basa en dos premisas: en la especialización y división del trabajo y en que las organizaciones se ven sometidas a un ambiente hostil que las obliga a mantener una cierta consistencia interna para enfrentarlo. La división del trabajo es entendida como un proceso descentralizador mediante el cual se implementa la política pública, presentando una apariencia unitaria. En este escenario y en el contexto de la implementación, el poder de decisión pasa del ápice estratégico a los directivos divisionales, pero el modelo de división del trabajo no se agota en la descentralización del poder.

La especialización del trabajo y la coordinación entre labores especializadas, son dos factores fundamentales para dar forma a la estructura de una organización. La propuesta de Mintzberg acerca de los distintos tipos de estructura organizacional, se basa en las distintas maneras como pueden coordinarse las actividades especializadas, esto es: estandarización de procesos, resultados o habilidades, supervisión directa y ajuste mutuo entre quienes cooperan.

Las estrategias diseñadas por las organizaciones, deben poseer parámetros que dependerán de las circunstancias. La primera serie de parámetros está asociada al diseño de las posiciones individuales dentro del programa: la especialización del trabajo, la formalización del comportamiento, la capacitación y el adoctrinamiento. La segunda serie de parámetros, se asocia a la red que genera subunidades: la agrupación de unidades y el tamaño de las unidades. La tercera serie de parámetros, está asociada a los vínculos o encadenamientos laterales usados para encarnar la superestructura: los sistemas de planeamiento y los dispositivos de enlace. El cuarto y último parámetro, está asociado con la toma de decisiones: la descentralización vertical y horizontal (Mintzberg et al., 1997). Sobre los parámetros influyen ciertos factores de contingencia, también llamados "factores situacionales", como la edad, el tamaño, el sistema técnico, el ambiente y el poder (Mintzberg, 1988).
Se añaden, como parte integrantes de la estructura, el sistema tecnológico e informático y los proveedores. Cada nivel será estudiado según una batería de indicadores también propuestos por Mintzberg (1988): mecanismos de coordinación, configuraciones, impulsos, parámetros, elementos situacionales, entorno y poder para dar respuesta a tres dimensiones: financiera, institucional y organizacional.

\section{Programas de Desarrollo Infantil Temprano (DIT) en Chile}

La política pública dirigida a la primera infancia en Chile, está compuesta de un conjunto diverso de programas. Entre estos se cuentan intervenciones de tipo sectorial, intersectorial, multisectorial e integral. La figura 1 presenta un inventario de los programas de DIT de Chile, según el tipo de intervención.

La totalidad de estas intervenciones se encuentran organizadas en el marco de la política pública Chile Crece Contigo (CHCC), cuyo eje es el programa de Apoyo al Desarrollo Biopsicosocial (PADBP), que realiza el seguimiento a la trayectoria de desarrollo de los niños desde el primer control prenatal, hasta su ingreso en el sistema escolar, alrededor de los 5 años de edad. Chile Crece Contigo, pertenece al Sistema de Protección Social denominado Subsistema de Protección Integral a la Infancia, dependiente del Ministerio de Desarrollo Social. Su misión institucional es contribuir al desarrollo del país con integración y protección social de las personas, mediante el análisis, diseño, coordinación, ejecución y evaluación de políticas sociales, planes y programas; la evaluación de las iniciativas de inversión pública; la provisión de información y análisis acerca de la realidad social y económica; y, la elaboración de instrumentos y metodologías para la gestión y toma de decisiones de políticas públicas (Gobierno de Chile, 2014).

En el marco del Sistema de Protección Integral a la Infancia, el Programa de Apoyo al Recién Nacido (PARN) comparte el fin de "potenciar el desarrollo integral de niños y niñas desde la gestación hasta su ingreso a pre kínder, para avanzar en la disminución de las brechas de desigualdad". Su propósito está definido como "aportar a que los niños y niñas nacidos en el sistema público de salud cuenten con las condiciones mínimas de bienestar que requieren para su desarrollo". Para efectos del Programa, se han definido las siguientes condiciones mínimas: i) lactancia materna exclusiva al menos hasta el sexto mes de vida; ii) proteger el sueño regular y facilitar condiciones para un dormir seguro; iii) proveer experiencias tempranas de estimulación 
Cuadro 1: Descripción de dimensiones

\begin{tabular}{|c|c|c|c|c|}
\hline Dimensiones & Descripción & \multicolumn{3}{|c|}{ Niveles } \\
\hline Financiera & $\begin{array}{l}\text { Recursos económicos que permitan alcanzar objetivos me- } \\
\text { diante el trabajo profesional y ético, desarrollado en un en- } \\
\text { torno de aprendizaje con cumplimiento de metas y bienestar }\end{array}$ & & Macro & \\
\hline Institucional & $\begin{array}{l}\text { La institución como estructura relevante para lograr los ob- } \\
\text { jetivos propuestos y coadyuvar al funcionamiento institu- } \\
\text { cional }\end{array}$ & Macro & Meso & Micro \\
\hline Organizacional & $\begin{array}{l}\text { La organización como estructura relevante para conseguir la } \\
\text { misión institucional y que establece el sentido y la dirección } \\
\text { hacia donde se está avanzado, además dota de elementos } \\
\text { necesarios para el desarrollo de sus actividades y cumpli- } \\
\text { miento adecuado de sus funciones para lograr los objetivos } \\
\text { propuestos y coadyuvar al funcionamiento institucional }\end{array}$ & Macro & Meso & Micro \\
\hline
\end{tabular}

Fuente: Elaboración propia a partir de Mintzberg et al. (1997)

como puede ser hablarles, cantarles, jugar y otros; y iv) mantener una relación de cuidados con personas significativas y estables en tiempo (Dirección de Presupuestos, 2011).

Para el logro de sus objetivos, el PARN cuenta con dos componentes: i) Componente set de implementos, que consiste en la entrega, en forma gratuita, de un set de implementos para los niños y niñas nacidos en hospitales del sistema público de salud, que se efectúa al momento del alta de las madres de las maternidades de los hospitales públicos; y, ii) Educación sobre uso de implementos y cuidados básicos de crianza temprana. Este último se materializa a través de dos modalidades: i) entrega de materiales educativos; y ii) sesiones educativas que se realizan en consultorios de Atención Primaria de Salud (APS) durante los controles prenatales y en los hospitales públicos, en forma previa al alta de las madres.

El PARN se vincula con el Programa de Apoyo al Desarrollo Biopsicosocial (PADBP) dependiente de la Subsecretaría de Redes Asistenciales del Ministerio de Salud (MINSAL) también inserto en el CHCC, con la Subsecretaría de Salud Pública y con el Sistema Intersectorial de Protección Social (SPS) que institucionaliza el Subsistema Chile Crece Contigo (CHCC), del Ministerio de Planificación Nacional (MIDEPLAN). Es un programa de ámbito nacional y su ejecución se inició en septiembre de 2009 por un período de tiempo indefinido (Dirección de Presupuestos, 2011).

\section{Metodología}

Esta investigación tiene un enfoque de tipo exploratorio (Barrantes Echavarría, 1999). Para el análisis se trabaja con el Modelo organizacional propuesto por Mintzberg, Quinn y Voyer (1997) y Mintzberg (1988). En base a esta concepción de estructura, flujos y tipología organizacional, se trabajará en torno a tres dimensiones: financiera, institucional y organizacional.

Se trabajará la estructura del PARN en seis niveles: el ápice estratégico, ocupada por el MINSAL y el MDS; la línea media, ocupada por las divisiones, el staff de apoyo y la tecno estructura; y el núcleo operativo, ocupado por los hospitales y atención primaria. En suma, se describe los niveles macro, meso y micro de la implementación del programa. Se añaden como parte integrantes de la estructura el sistema tecnológico e informático y los proveedores. Cada nivel será estudiado según una batería de indicadores también propuestos por Mintzberg: mecanismos de coordinación, configuraciones, impulsos, parámetros, elementos situacionales, entorno y poder.

En su aspecto cualitativo, se trabajó con información secundaria extraída de los documentos institucionales, de las evaluaciones y de otras fuentes de información; y, de información primaria, mediante la realización de entrevistas con informantes calificados. De esta forma, se desarrolla una estrategia de triangulación, de manera de aumentar la capacidad analítica, la fiabilidad y la validez del estudio. Se elaboró una pauta de entrevista, la que se estructuró en una serie de módulos y se articuló bajo dos modalidades. Una modalidad, que contiene una serie de preguntas abiertas con el objetivo de motivar 
Figura 1: Programas Desarrollo Infantil Temprano en Chile por tipo.

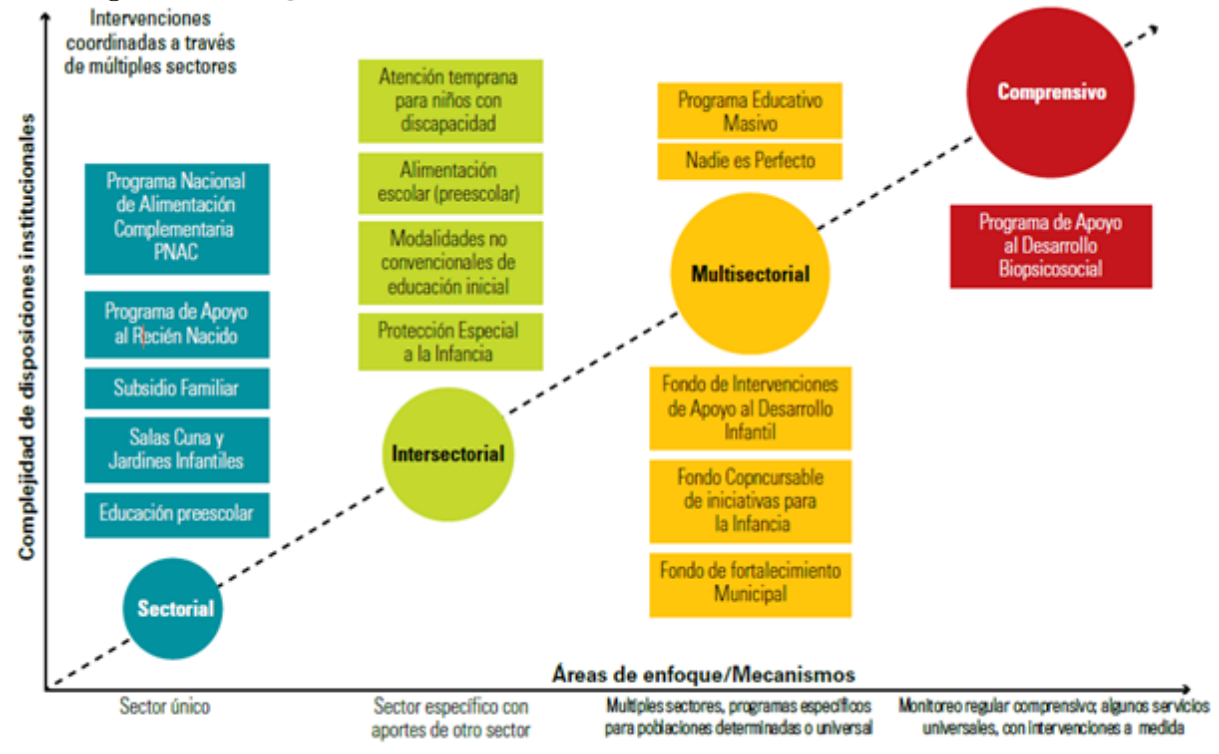

Fuente: Banco Mundial (2011)

el discurso del entrevistado y lograr su percepción sobre temas claves, de manera de construir la realidad del PARN.

Por otra parte, para el análisis cuantitativo, en esta misma entrevista, se incluyeron una serie de tópicos asociados al PARN, sobre los cuáles se le pidió al entrevistado su evaluación, haciendo uso de una Escala Likert de cinco puntos, permitiendo así el análisis cuantitativo. Las entrevistas fueron grabadas, transcritas y codificadas, y de estricto uso confidencial para la presente investigación. Las preguntas con respuesta cerrada del tipo dicotómicas, o que hacen uso de la escala Likert, exigieron el armado de una estructura de base de datos haciendo uso de un software libre conocido como Epidata. Una vez ingresadas las respuestas, la base fue exportada al paquete estadístico SPSS, mediante el cual se hizo el procesamiento de los datos. Para un mayor desempeño de la información generada en esta fase, las respuestas obtenidas haciendo uso de la escala Likert de cinco puntos, fueron integradas en dos categorías, bajo las opciones de respuesta "de acuerdo" y "en desacuerdo", según la respuesta.

La fase de campo fue realizada entre los meses de junio, julio y agosto del 2014. Las entrevistas operativamente se realizaron cara a cara en el caso de los informantes de Santiago y con apoyo tecnológico para conversación a distancia para aquellos situados en Regiones.

\section{Resultados}

El Programa de Apoyo al Recién Nacido, nace de la necesidad de cooperar con los sectores más vulnerables de la población. Dos factores fundamentales intervienen en toda su estructura: por un lado, la división del trabajo en distintas áreas, entendida como una separación de actividades con el fin de lograr una mayor precisión, eficiencia y especialización de cada una de las mismas; y por otra parte, la coordinación, que es la sincronización de los esfuerzos. Estos dos factores, definen al PARN en su estructura y como organización.

El mecanismo más frecuente para la coordinación de tareas utilizado en el PARN, es el de las comunicaciones formales entre quienes lo integran. Esto de alguna manera habla de que existe un sistema integrado de información. Muchas veces, estas comunicaciones formales de coordinación de tareas, son antecedidas por una comunicación informal.

Si bien los entrevistados reconocen que en el PARN están presentes todos los mecanismos consultados para coordinar las tareas del Programa, existe una clara tendencia a mencionar los mecanismos de "comunicación formales" como los más frecuentes para coordinar las tareas del PARN. Si bien las categorías propuestas no son excluyentes, el $83,3 \%$ de los entrevistados indican a este mecanismo como frecuente o muy frecuente; seguida de las "metas a alcanzar", con un $66,7 \%$ de menciones como frecuente o muy frecuente.

Los dispositivos de coordinación que utiliza en la 
Figura 2: Mecanismos de coordinación

-Nada, poco $\| \mathrm{Ni} / \mathrm{Ni} \quad$ || Frecuente, muy $\| \mathrm{NS} / \mathrm{NC}$

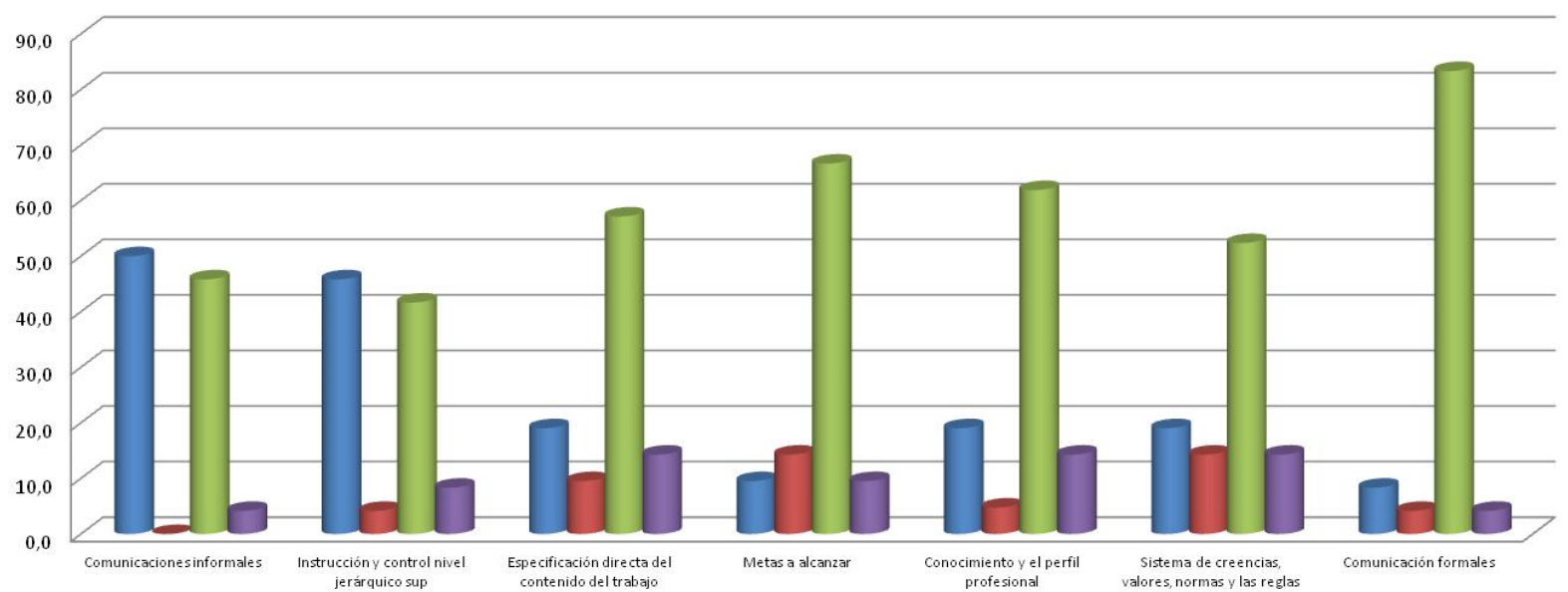

Fuente: Elaboración propia

actualidad el Programa son valorados positivamente. Un $79,2 \%$ de los entrevistados señala como muy adecuados y adecuados los dispositivos de coordinación. Por tanto, no se trata de una organización con lo que Mintzberg llama "ajuste mutuo", ni por "supervisión directa", sino que está basada en la estandarización de los procesos de trabajo, de los resultados y de las destrezas o habilidades de sus trabajadores.

En esta unión de procesos, resultados y especialización de habilidades, los entrevistados entienden que los dispositivos de coordinación implementados son adecuados o muy adecuados. Casi un $80 \%$ de los consultados responden que son adecuados o muy adecuados los mecanismos de coordinación del Programa, mientras que un porcentaje ínfimo entiende como poco adecuados o nada adecuados los mecanismos de coordinación. De algún modo, quienes integran el programa, tienden a preferir la formalización de las tareas con el fin de reducir su variabilidad, predecir, controlar, coordinar actividades y obtener mayor eficiencia, a través del aseguramiento de la consistencia mecánica, que redunda en asegurarle el beneficio a las madres y a los recién nacidos.

Con el objetivo de dar forma a la configuración de la estructura que conforma el PARN como organización, se les planteó a los entrevistados varias opciones de configuración organizacional, de manera de aproximarse a la idea que construyen del Programa. El Programa tiende a ser definido como una estructura "sencilla y flexible", "estandarizada en la tarea", "profesionalizada", y, en menor medida "diversificada en divisiones intermedias" y "unida". Se trata de una organización que modela una estructura organizacional del tipo burocrática profesional, con mucho poder de "hacer" en el centro operativo, formado por profesionales altamente capacitados.

Los integrantes del PARN, visualizan el diseño organizacional de la institución con respecto al trabajo del personal, como "especializado". Un 71,4\% de los entrevistados señalan como muy especializado o especializado el trabajo del personal del Programa.

El PARN, se percibe como una organización con alta formalización de los comportamientos del personal que lo integra. Un $75 \%$ de los entrevistados señala como muy formalizado o formalizado el comportamiento de su personal. La agrupación de la red de unidades, es considerada como adecuada o muy adecuada por el $81 \%$ de los entrevistados. Sin embargo, el tamaño de las agrupaciones es considerado como pequeño para casi el $43 \%$ de los entrevistados.

Los entrevistados consideran que los sistemas de planificación son muy adecuados o adecuados. Probablemente este grado de adecuación de los sistemas de planificación permea sobre el grado de formalización de las tareas. En la toma de decisiones intervienen distintas alternativas que involucran factores económicos, técnicos, humanos y sociales. En el ámbito del PARN, tomar una decisión implica elegir aquella alternativa coincidente con la optimización del valor social que genera el Programa. La descentralización o centralización del Programa puede entenderse como un medio cerrado o abierto de coordinación en el cual se toman las decisiones. Estas, pueden ser tomadas desde el centro y luego ser implementadas a través de lo que podría 


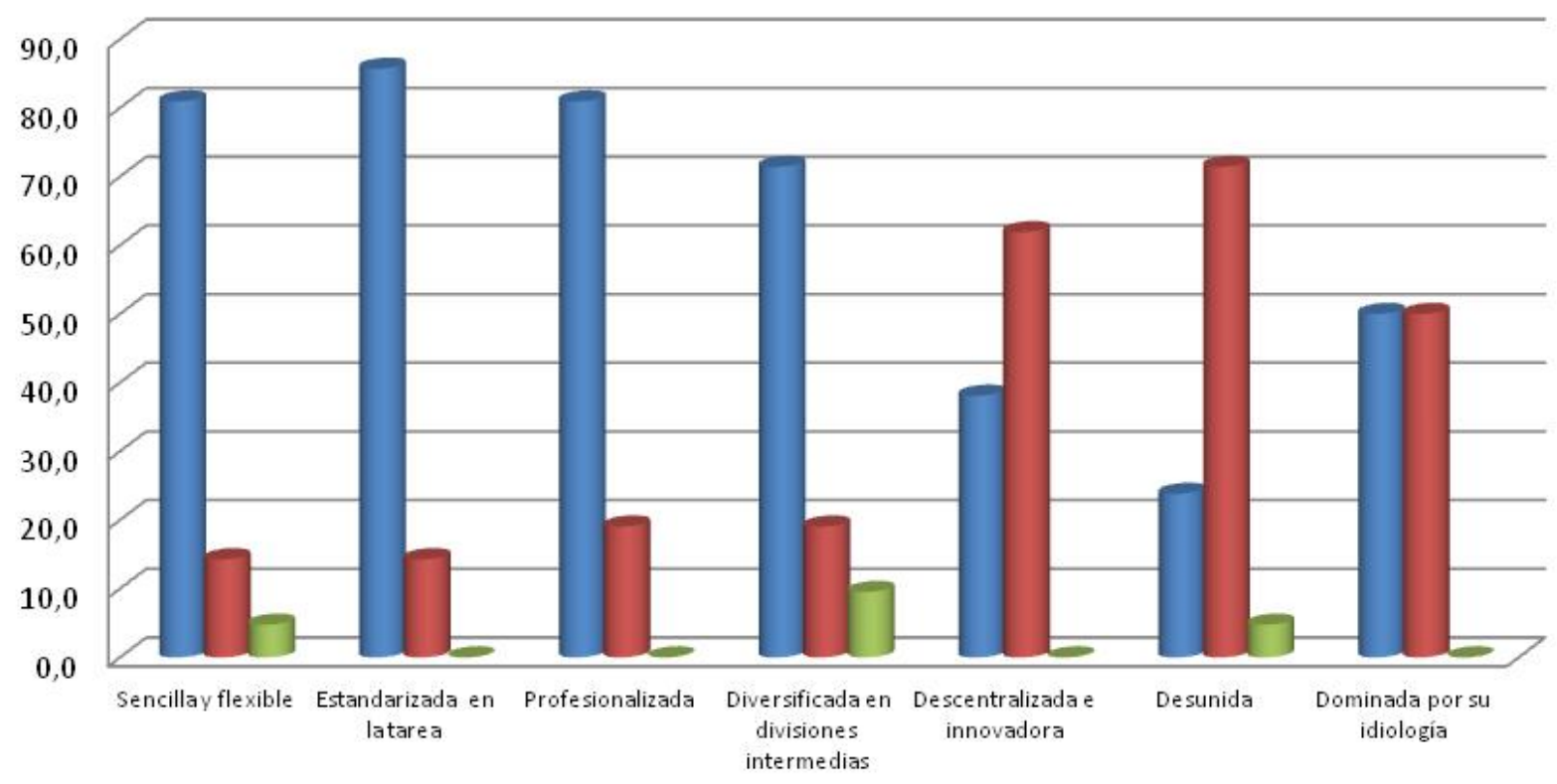

Fuente: Elaboración propia

entenderse como una supervisión directa. Como la estructura del Programa no es demasiado grande, las decisiones pueden ser fácilmente tomadas a nivel central y la información re transmitida a los restantes niveles de manera comprensible por quienes están en contacto diario con la realidad del Programa y sus beneficiarios inmediatos. La mirada de los entrevistados, refleja una estructura centralizada en la toma de decisiones. Esto podría generar una estructura que no favorece la rápida respuesta a cambios en las condiciones a nivel local, no beneficiándose del conocimiento de este nivel y desmotivando al personal.

Es decir, el PARN se percibe como una estructura centralizada y formal, donde el control externo sobre el Programa es alto. Este poder mayor tiende a alentar las formas formalizadas y reguladas, mediante un sistema de normas, reglas y regulaciones. Esto le resta autonomía al nivel de las regiones, lo que sin dudas es percibido por las mismas como una desventaja para el buen funcionamiento del Programa.

A pesar de tratarse de una estructura altamente profesionalizada, los profesionales involucrados perciben que su trabajo es controlado desde el exterior y normalizado por la Dirección del PARN.

Con respecto a los elementos situacionales del PARN, los entrevistados coinciden en que el transcurso del tiempo ha proporcionado un grado de aprendizaje que le ha permitido introducir mejoras en los procesos, fundamentalmente asociado con los proveedores. El $57 \%$ de los entrevistados, perciben que el paso del tiempo ha influido de manera muy positiva o positiva sobre el Programa, formalizando su comportamiento.

De los entrevistados indagados sobre si el entorno político influye sobre el Programa, casi el $62 \%$ afirma que influye poco o muy poco. Esto da cuenta de que el entorno externo en el que se desarrolla el PARN, que le proporciona estabilidad.

Sin embargo, al consultar a los entrevistados si existieron cambios en el PARN atribuidos a los cambios de gobierno, más de la mitad de los entrevistados responden que existieron muchos cambios debido a la sucesión en el gobierno ${ }^{1}$.

La planificación y el control pueden entenderse como los ámbitos reguladores del comportamiento y de las acciones específicas del PARN, estando íntimamente encadenas una a la otra. Planificar en el ámbito del Programa es prever y decidir hoy las acciones que lleven a un futuro deseable, lo que está estrechamente ligado a la toma de decisiones.

La planificación está orientada a las actividades específicas que desarrolla el PARN. Sin embargo, el control del desempeño que mide el resultado en el tiempo, regula la unidad de trabajo y mide desem-

\footnotetext{
${ }^{1}$ Desde el primer gobierno de centroizquierda de Michelle Bachelet (2006-2010) al gobierno de centroderecha Sebastián Piñera (2010-2014).
} 
Figura 4: Agrupación de unidades por tamaño del PARN

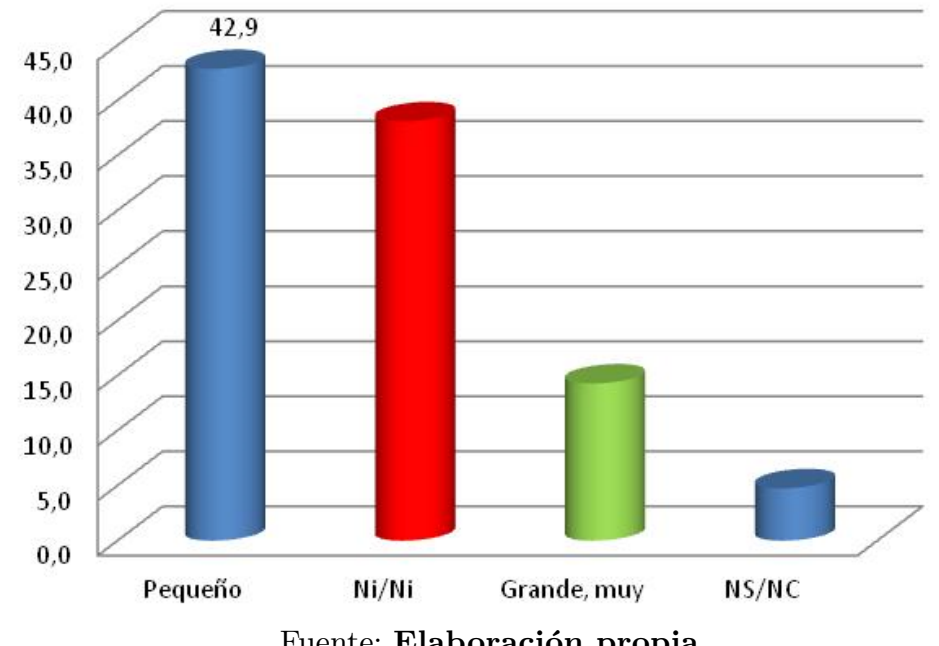

Figura 5: Influencia de los años sobre el PARN

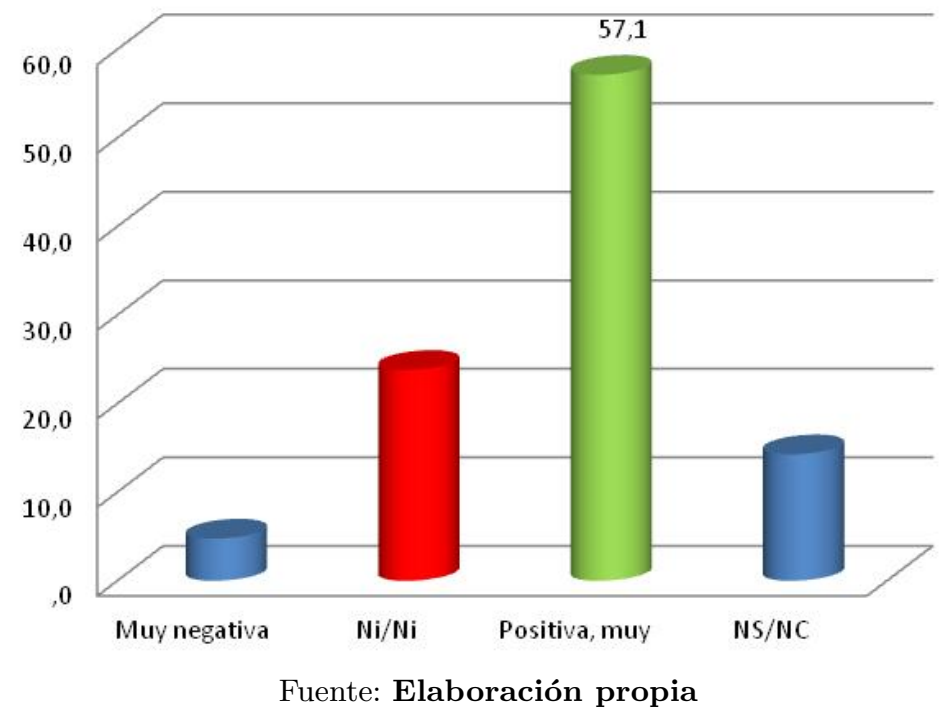

peño del Programa, se realiza en función del cumplimiento de lo previamente establecido.

Indagados sobre si consideran que en el PARN se aplican pocos o muchos mecanismos de control, el $80 \%$ de los entrevistadores coinciden en que se aplican o se aplican muchos mecanismos de control.

El nivel de cooperación entre quienes integran el Programa es considerado extremadamente elevado. Se trata de una organización en la cual la cooperación entre los distintos niveles funciona. El $91 \%$ de los entrevistados señala como muy cooperantes o cooperantes a los distintos actores comprometidos en el PARN.

Los amiguismos políticos, que muchas veces son un factor determinante en los procesos de una organización o institución, no son considerados un incentivo para la gerencia del PARN. El 71,4\% de los entrevistados indican que este influye poco o nada en la gerencia del PARN.

En su burocracia profesional, la estandarización de destrezas no es observable, ya que estas son adquiridas fuera del PARN. Sin embargo, el núcleo operativo mantiene una comunicación directa con los usuarios del Programa. El PARN es percibido como una organización que contiene a personal muy capacitado. Un 87,5\% de los entrevistados sostienen que su personal está muy capacitado o capacitado. Este alto nivel de capacitación del personal asociado al PARN, contrasta bastante con la percepción de la capacitación que el Programa ofrece a sus recursos humanos en forma directa. Un alto porcentaje de los entrevistados percibe como poco o nada adecuada la capacitación brindada, mientras también otro alto porcentaje percibe la capacitación como adecuada o muy adecuada. 
Figura 6: Grado de influencia del entorno político sobre el PARN

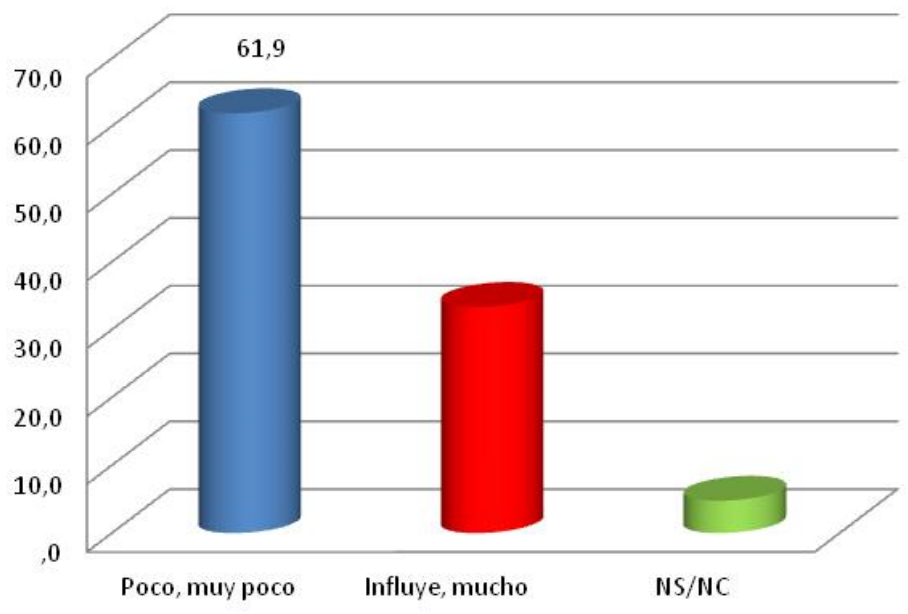

Fuente: Elaboración propia

Figura 7: Nivel de capacitación que brinda el PARN a sus RRHH (\%)

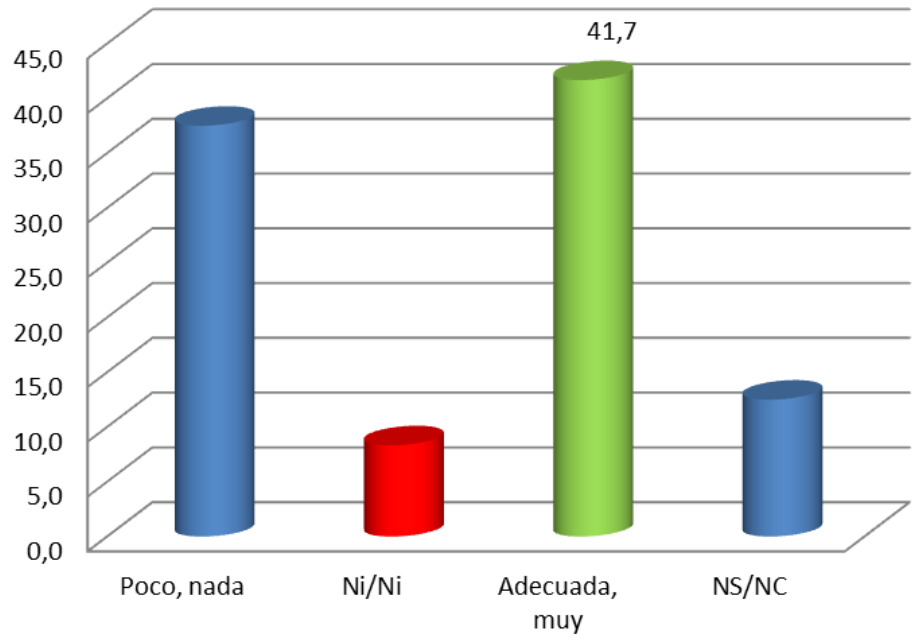

Fuente: Elaboración propia

Siguiendo el modelo de Mintzberg y a la luz de la información recogida, se puede observar la forma que adquiere la estructura del PARN, asociada a lo que el autor describe como una "estructura profesional"

Pese a que los entrevistados identifican al PARN con una estructura planificada, y en donde se aplican muchos mecanismos de control, al tiempo que es profesionalizada y especializada, las decisiones que se toman no son percibidas como decisiones informadas a través de un sistema informático. Ante la pregunta de si considera que la información que proporcionan los sistemas informáticos del PARN le permite al Programa tomar decisiones en tiempo real, el 58,3\% de los encuestados, responde que esas decisiones son tomadas de forma poco o nada informadas.
Esta situación, se debe a que el PARN carece de un sistema informático integrado y transparente. Los distintos actores que participan del Programa tienen accesos diferenciales a las bases de datos y muchas veces existe rezago en la completitud de las mismas, debido especialmente a la ausencia de personal directamente afectado a esta función. Al no utilizarse el soporte informático como alternativa de resolución en la toma de decisiones, se deben tomar decisiones poco informadas, artesanales, que siguen las variaciones y expresiones del operario. A pesar de la falta de un sistema informático que permita tomar decisiones en tiempo real, un 72,9\% de los entrevistados considera que existe una resolución compartida de problemas.

Con respecto al comportamiento de la Dirección del PARN, los entrevistados coinciden mayoritariamente en que la Dirección está abocada a la búsque- 
da de nuevas oportunidades de manera de aumentar el valor social que genera el Programa. El 79.2\% está de acuerdo con el carácter innovador que intenta imprimirle la Dirección al Programa.

Analizando el discurso de los entrevistados, es posible identificar el grado de acuerdo entre ellos sobre el objetivo principal que persigue el Programa de Apoyo al Recién Nacido. Se tiende a considerar el objetivo del PARN, no como un objetivo instrumental, por lo contrario, es visualizado como parte integrante de un esfuerzo desde el Estado hacia a la construcción de una sociedad más justa. Los profesionales implicados en el Programa, entienden que es una política de Estado que trasciende a los gobiernos y que su objetivo es beneficiar a los sectores más vulnerables de la sociedad chilena. "El objetivo principal es dar igualdad [...] que todos los niños que nazcan en el sector púbico, nazcan en las mismas condiciones. En el sentido de que puedan acceder a una infancia justa" (Analista presupuestario, DIPRES).

Para alcanzar este objetivo, la estructura del Programa se presenta como estructura diversificada de roles, que colaboran, integrada por varias instituciones, bajo la lógica de la intersectorialidad. En ese sentido, participa el Ministerio de Desarrollo Social, el Ministerio de Salud y su Red de Hospitales Públicos, la CENABAST, operadores logísticos, proveedores y DIPRES. Bajo esta estructura intersectorial, se planifica, coordina, controla, administra y abastece el Programa.

Los recursos están en el presupuesto del Ministerio de Desarrollo Social, específicamente en el programa presupuestario que se llama Sistema Integral de Protección a la Infancia [...] esos recursos año a año son entregados y validados en el momento que se empieza a discutir el presupuesto. La institución cada año a nosotros nos solicita, o una la base del presupuesto o incrementarse.

(Analista presupuestario, DIPRES)

La intersectorialidad es base constitutiva del sistema de protección chileno, y es entendida, por muchos de los entrevistados, como una necesidad intrínseca al abordaje del desarrollo infantil. Desde el punto de vista de la intervención es una ventaja en sí misma, ya que cuenta con la participación de múltiples actores, aportando cada cual sus capacidades y otorgándole al Programa una mirada más integrada.

La forma de trabajo que se ha instalado en el PARN es percibida como muy horizontal en todos los niveles entorno a un objetivo común, pero sin una exacta visualización de quién es el que toma las decisiones en última instancia. El nivel jerárquico del Programa se torna algo difuso.

Sin embargo, la participación de muchos actores e instituciones genera dificultades en la toma de decisiones, que implica ajustes mutuos en los tiempos, reglas de cada institución y procesos de adaptación a las burocracias particulares de cada nivel de la estructura. En esta dinámica intersectorial, muchas veces el tratamiento de los temas queda supeditado a la buena voluntad de las "personas". La gran dificultad, es integrar la mirada intersectorial más allá de la mirada de conjunto, una mirada de trabajo en "equipo", que a pesar de los esfuerzos, no existe.

Si bien, el Programa se ha ido perfeccionando a medida que pasan los años, se observa la existencia de cuellos de botella que dificultan la implementación del Programa y por tanto disminuyen el nivel de eficiencia y eficacia del mismo. Estos cuellos de botella de la implementación se dan fuertemente a nivel de la red hospitalaria en temas fundamentalmente de educación y registro. El registro es señalado como una carencia grave para la implementación del PARN. Asimismo, el desconocimiento con el que llegan las usuarias del Programa, genera, en sí mismo, una dificultad porque no se visualiza como un componente educativo del mismo.

La necesidad de contar con sistemas de registro eficientes, es visualizada por los entrevistados como alineada con la necesidad de introducir un sistema informático potente, que permita la toma de decisiones informadas en tiempo real.

Hoy día todavía tenemos un margen de mejoramiento asociado a cómo logramos que los hospitales pudieran estar en línea para la solicitud de su manejo de stock y como todo lo que tiene que ver con esa parte de entrega y recepción estuviera mucho más informatizada.

(Profesional de apoyo CHCC, MDS)

También se señala cierta dificultad con respecto a las temáticas más operativas de la implementación, asociadas a la adecuación del espacio de los hospitales para el stock y la falta de personal específicamente comprometido a nivel de bodegas del hospital con el PARN. 
Figura 8: Comportamiento de la dirección del PARN

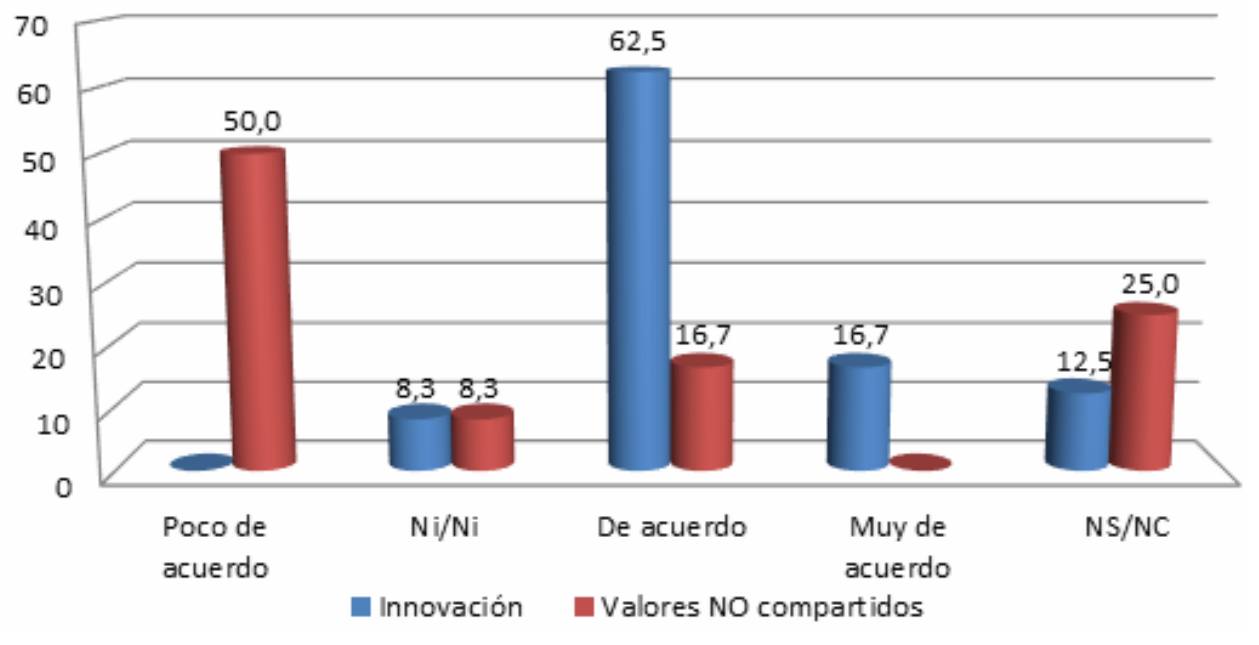

Fuente: Elaboración propia

Creo que en general a nivel de los interlocutores que dirigen y coordinan el programa, es fluida y no hay mayor dificultad. Yo diría que la dificultad se produce en el punto de destino, donde hay una persona que está viendo su realidad personal y probablemente no tiene la mirada del Programa como beneficio para su institución.

(Biomedical Distribution, Operador Logístico)

Otra dificultad en la implementación, está asociada con los convenios y los tiempos burocráticos que insumen los procesos de compra con el Estado. Esta situación afecta fundamentalmente al operador logístico y proveedores, como consecuencia de los procesos asociados a las compras y licitaciones públicas cuyo actor principal es CENABAST.

El análisis de las entrevistas realizadas, permiten identificar que el Programa de Apoyo al Recién Nacido como organización, se compone de dos factores: la división del trabajo en las distintas áreas y la coordinación que se debe lograr entre las mismas para alcanzar los objetivos que la organización persigue. Mediante la construcción de forma explícita y formal de la estructura del PARN y de la formalización de su comportamiento para lograr la coordinación, el Programa puede ser definido como una organización formalizada, profesionalizada y estandarizada.

La coordinación del PARN es un proceso que integra las actividades de los distintos actores involucrados, con la dificultad de la coordinación entre distintos sectores, para alcanzar las metas del Programa con eficacia. Los mecanismos de coordinación son necesarios porque de no existir, en un Programa intersectorial como el PARN, quienes lo integran perderían de vista sus roles dentro de la or- ganización. El grado de coordinación que existe en la actualidad depende de la naturaleza de las tareas en las que está implicado cada actor, institución y nivel local o central.

Los mecanismos de coordinación empleados en el PARN, son valorizados como eficientes para la coordinación de las tareas por sus trabajadores. De algún modo quienes integran el programa tienden a preferir la formalización de las tareas con el fin de reducir su variabilidad, predecir, controlar, coordinar actividades y obtener mayor eficiencia, a través del aseguramiento de la consistencia mecánica, que redunda en asegurarle el beneficio a las madres y a los recién nacidos. La comunicación entre los distintos estamentos dentro del PARN tiende a estar formalizada.

A nivel de la coordinación, los principales cuellos de botella están asociados con las redes hospitalarias. Los Directores no tienen un rol definido en el Programa y el Programa es asumido por las autoridades del Hospital como un beneficio que otorga CHCC. Principalmente CENABAST, y en menor medida los proveedores, son señalados como los actores con los cuáles se torna más dificultosa la coordinación. La rotación de personal, también es señalada como una dificultad a la hora de coordinar las actividades del Programa.

Los procesos de planificación, no comprometen a la totalidad de los miembros del Programa, debido que la misma se da en un ámbito acotado y centralizado.

Existe la percepción de los entrevistados, que el PARN posibilitó a la Red de Hospitales y al sistema integral del Chile Crece Contigo, mejorar la calidad de la relación con los usuarios, sobre todo a nivel 
local.

Acompañando a una cierta mística que se dice tiene el PARN, los entrevistados coinciden en que el Programa debe de tener continuidad y seguir apostando a mejorar todas y cada una de sus instancias.

Considero que el Estado chileno tiene que avanzar, no sólo aquí, sino en muchas otras áreas en la garantía de derecho y protección universal. Este es uno de los pocos programas que establece una prestación que llegue al cien por ciento de los niños que se atienden en el sistema público sobre todo por las condiciones o las necesidades a las que está apuntando. Si tuviéramos mucho más claridad o mucho más consciencia de la importancia de eso en el desarrollo del capital humano de un país, creo que estaríamos hablando de otra situaciones de desarrollo. Me parece que no hay mejor inversión de aquella que se hace en la infancia, y estos son elementos que apuntan en esa línea. Entonces, por supuesto que tendría que tener continuidad y por supuesto que debería invertirse más plata en la parte educativa

(Profesional de apoyo CHCC, MDS)

\section{Conclusiones}

La temática de la infancia, es particularmente sensible para quienes trabajan en la salud y se reviste de una importancia que trasciende la esfera meramente instrumental y laboral. Pasa a ser considerada un imperativo, unido a la vocación social de servicio.

El modelo de Mintzberg que sostiene esta investigación, es un enfoque multifacético y complejo, que nos ha permitido analizar, clasificar, comprender y diagnosticar al Programa de Apoyo al Recién Nacido. El estudio de su estructura, nos ha permitido arribar a la conclusión de que su estrategia está vinculada con su diseño de la organización, lo que le ha permitido enfrentar exitosamente el desafío de la eficiencia y la eficacia a través del tiempo y los diferentes entornos que ha transitado desde su creación.

Desde esta perspectiva teórica, fue posible identificar en el Programa de Apoyo al Recién Nacido dos componentes ineludibles a toda organización, coincidentes con el modelo organizacional propuesto por Mintzberg: por un lado, la división del trabajo en distintas áreas; y por otro, la coordinación que se debe alcanzar para el logro de los objetivos que el mismo como política persigue.

El Programa de Apoyo al Recién Nacido, es lo que se entiende como una organización profesionalizada, una burocracia que no cuenta con el nivel de centralización de la burocracia mecánica. El trabajo profesional es complejo, pero estandarizado en las habilidades o destrezas de sus integrantes, garantizando que los profesionales trabajen en el núcleo de operaciones con igual grado de conocimiento y las mismas competencias nucleares. Esta situación es posible, porque el PARN se inserta en un medio ambiente estable, pero complejo, que si bien mantiene la toma de decisiones centralizada, aboga por una mayor descentralización para trabajar con un grado de autonomía más elevado. Esta configuración, depende de las habilidades de su personal y no de los procesos de trabajo o de los resultados para su coordinación. En el PARN, predomina el impulso hacia la profesionalización, dependiendo de profesionales capacitados y especializados que desempeñan sus tareas operativas en una estructura horizontal, aunque no descentralizada como lo es este tipo de estructura. Las decisiones descienden del nivel jerárquico superior hacia el núcleo de operaciones fuertemente centralizadas.

Los elementos cruciales que definen el Programa son: la formalización, la profesionalización y la estandarización de la tarea y las destrezas. La principal estandarización del PARN ocurre como resultado de la capacitación y destrezas de su personal.

En su burocracia profesional, la estandarización de destrezas no es observable, ya que estas destrezas son adquiridas fuera del PARN. Sin embargo, el núcleo operativo mantiene una comunicación directa con los usuarios del Programa y de esta manera indirecta muestra su importancia.

La edad, tiene efecto sobre la estructura en tanto le ha dado experiencia en los procesos y los ha fortalecido, formalizando su comportamiento. El medio ambiente en el cual se inserta el Programa, a pesar de su complejidad, es estable. Esta estabilidad, es la que le permite aplicar a los profesionales habilidades estandarizadas y trabajar con cierta autonomía.

El Programa de Apoyo al Recién Nacido, se basa en la formalización de su comportamiento, el control, la planificación, la profesionalización y la estandarización. En términos de Mintzberg, es susceptible de ser definido como una "organización de desempeño" y no de "resolución de problemas".

Existe un grado de acuerdo entre quienes lo integran, en definir como el objetivo principal que persigue el Programa asociado fuertemente a parámetros de equidad e igualdad social y en su contribución a los derechos de la infancia.

El carácter intersectorial del Programa, le da una visión integral en concordancia con el sistema de 
protección chileno. La misma, guarda como ventaja que la focalización de la población objetivo posee múltiples abordajes.

El PARN, también se presenta como una estructura profesional en cuanto a la estandarización de sus destrezas, existiendo además de estandarización, especialización y capacitación de los profesionales que integran su estructura. En el otro extremo, se encuentran los proveedores, donde el principal mecanismo de coordinación es el ajuste mutuo pues la formalización del comportamiento es baja y no existe una adecuada estandarización para una mejor coordinación. Los objetivos del PARN y de los proveedores, si bien han tendido a mejorar su ajuste, no se encuentran plenamente alineados. El PARN, con el paso del tiempo y la experiencia, ha buscado estandarizar los procesos, teniendo como cuello de botella principal entre el Programa y los distribuidores, el rol de CENABAST.

Según se desprende del discurso de varios de los participantes, la creación y operación de instancias de encuentro entre distintos sectores, es clave para el funcionamiento del Programa. Sin desmedro de ello, las percepciones varían ostensiblemente cuando se pregunta sobre la eficacia de las diferentes instancias.

El desafío deseable para el Programa de Apoyo al Recién Nacido, es profundizar y fortalecer la entrega y contenidos del componente educativo de Programa, continuar mejorando la relación con los usuarios, niños, niñas y familias de todo Chile, mediante la introducción de mejoras específicas en los procesos de implementación y coordinación del Programa.

\section{Recomendaciones}

Finalmente, este estudio de caso permite arribar a propuestas para fortalecer el Programa en sus niveles macro, meso y micro, a partir de los hallazgos previamente analizados. Los cambios que se proponen, se basan principalmente en aspectos relacionados con la implementación y coordinación del Programa. Al tratarse de un Programa relativamente nuevo en el tiempo, se pueden llevar a cabo cambios que mejoren sus niveles de implementación y coordinación, de manera de no afectar una cultura organizacional ya instalada.

De esta forma, las principales recomendaciones que se desprenden de la investigación presentada son:

- Potenciar el módulo educativo a través del for- talecimiento de los talleres pre natales.

- Introducir un sistema de registro potente, eficiente, que no admita brechas ni desfasajes en la información.

- Tener personal a nivel de la Red Hospitalaria directamente asociado al Programa.

- Evitar los procesos de rotación de personal, a través de medidas de contratación más atractivas de manera de contar con recursos humanos capacitados en las especificidades del Programa.

- Dotar de presupuesto adicional a los equipos locales, para poder hacer frente a las distintas necesidades. Apoyo a los niveles locales, mayor difusión del programa y del beneficio del uso de ciertos implementos, intensificar la educación.

- Buscar mecanismos que tiendan a darle una mayor visibilización de los recursos humanos, principalmente a nivel de la Red Hospitalaria.

- Contar con un sistema informático robusto y transparente, accesible a todos los niveles de la estructura, que permita la toma de decisiones en tiempo real informadas.

- Protocolizar de mejor manera el trabajo intersectorial, es decir, determinar en qué época del año se hace una evaluación, que participen todos los actores, que puedan modificarse aquellos aspectos que los distintos actores les parece relevante modificar.

- Modelar, valga la redundancia, el modelo de gestión.

- Generar una memoria institucional que permita la transmisión de conocimiento de unos a otros y de un período a otro.

- Readecuar el Programa contemplando plazos de convenios más amplios, para alinear los objetivos del PARN con los de sus proveedores.

- Mejorar los canales de comunicación entre el Programa y los proveedores, de forma de atender sus sugerencias y reclamos e implementar mejoras necesarias para el éxito del Programa.

- Incentivar la contratación de personal exclusivamente dedicado al Programa a nivel de la Red Hospitalaria.

\section{Referencias}

Banco Mundial (2011). Fortalecimiento de Políticas Públicas y Desarrollo Infantil Temprano en América Latina y el Caribe. 
Barrantes Echavarría, R. (1999). Investigación: un camino al conocimiento: un enfoque cualitativo y cuantitativo. EUNED, San José, Costa Rica.

Cortázar Velarde, J. C. (2006). Una mirada estratégica y gerencial de la implementación de los programas sociales. En Entre el Diseño y la Evaluación. Inter-American Development Bank, Washington DC.

Cunill-Grau, N. (2014). La intersectorialidad en las nuevas políticas sociales: Un acercamiento analítico-conceptual. Gestión y política pública, 23(1):5-46.

Dirección de Presupuestos (2011). Evaluación al Programa de Apoyo al Recién Nacido. Informe Final.

Echebarría, K. (2008). El papel de la profesionalización del empleo público en América Latina. En Longo, F. y Ramió, C., editores, La profesionalización del empleo público en América Latina. Fundació CIDOB, Barcelona.

Gobierno de Chile (2014). Definiciones Estratégicas Ministeriales 2010-2014. Formulación Presupuestaria 2013.
Licha, I. y Molina, C. G. (2006). Coordinación de la Política Social: criterios para avanzar. Serie de Documentos de Trabajo. Departamento de Integración y Programas Regionales, Instituto Interamericano para el Desarrollo Social, Banco Interamericano de Desarrollo.

Martínez, R. (2010). La coherencia y la coordinación de las políticas públicas. Aspectos conceptuales y experiencias. En Acuña, C., editor, Los desafíos de la coordinación y la integralidad de las políticas y gestión pública en América Latina., número 35 (8). Jefatura de Gabinete de Ministros, Buenos Aires.

Mintzberg, H. (1988). La estructuración de las organizaciones. Ariel, Barcelona.

Mintzberg, H., Quinn, J. B., y Voyer, J. (1997). El proceso estratégico: conceptos, contextos y casos. Pearson Educación.

Peters, B. G. (1998). Managing Horizontal Government: The Politics of Coordination. Public administration, 76(2):295-311. 